\title{
BONE-INSPIRED 3D PRINTED STRUCTURES FOR
} CONSTRUCTION APPLICATIONS

\author{
Roberto Naboni' ${ }^{1}$, Anja Kunic ${ }^{1}$
}

ABSTRACT: Overconsumption of resources is one of the greatest challenges of our century. The amount of material that is being extracted, harvested and consumed in the last decades is increasing tremendously. Building with new manufacturing technology, such as 3D Printing, is offering new perspectives in the way material is utilized sustainably within a construction. This paper describes a study on how to use Additive Manufacturing to support design logics inspired by the bone microstructure, in order to build materially efficient architecture. A process which entangles computational design methods, testing of 3D printed specimens, developments of prototypes is described. A cellular-based tectonic system with the capacity to vary and adapt to different loading conditions is presented as a viable approach to a material-efficient construction with Additive Manufacturing.

KEYWORDS: Functionally Graded Trabecular Tectonics; Digital fabrication; Additive Manufacturing; Computational Design; Biomimetics. 


\section{INTRODUCTION}

\section{Material Scarcity}

Overconsumption of resources is one of the greatest challenges of $21^{\text {st }}$ century. The tremendous increase in the amounts of material that is being extracted, harvested and consumed in the last decades is bringing to the serious problem of material and resource scarcity (Fig. 1). Coal, oil and gas are not only becoming increasingly rare, but are also accelerating emergence of sensitive global crisis leading to climate change and exceeded $\mathrm{CO}_{2}$ emissions. Even though the most usual associations to the pollution and $\mathrm{CO}_{2}$ emission are cars and aeroplanes, these are not the crucial culprits. In fact, another area that is more critical and far more problematic is the building construction, which causes, directly or indirectly, $80 \%$ of $\mathrm{CO}_{2}$ emissions. Therefore, architects are challenged to rethink the way of designing, building and confronting with the of materials in the built environment. New strategies are needed to adapt to the post-fossil age and make a crucial contribution to the ecological turnaround through the intelligent and looking-forward design, use of materials, technologies, recycling requirements and energy use.

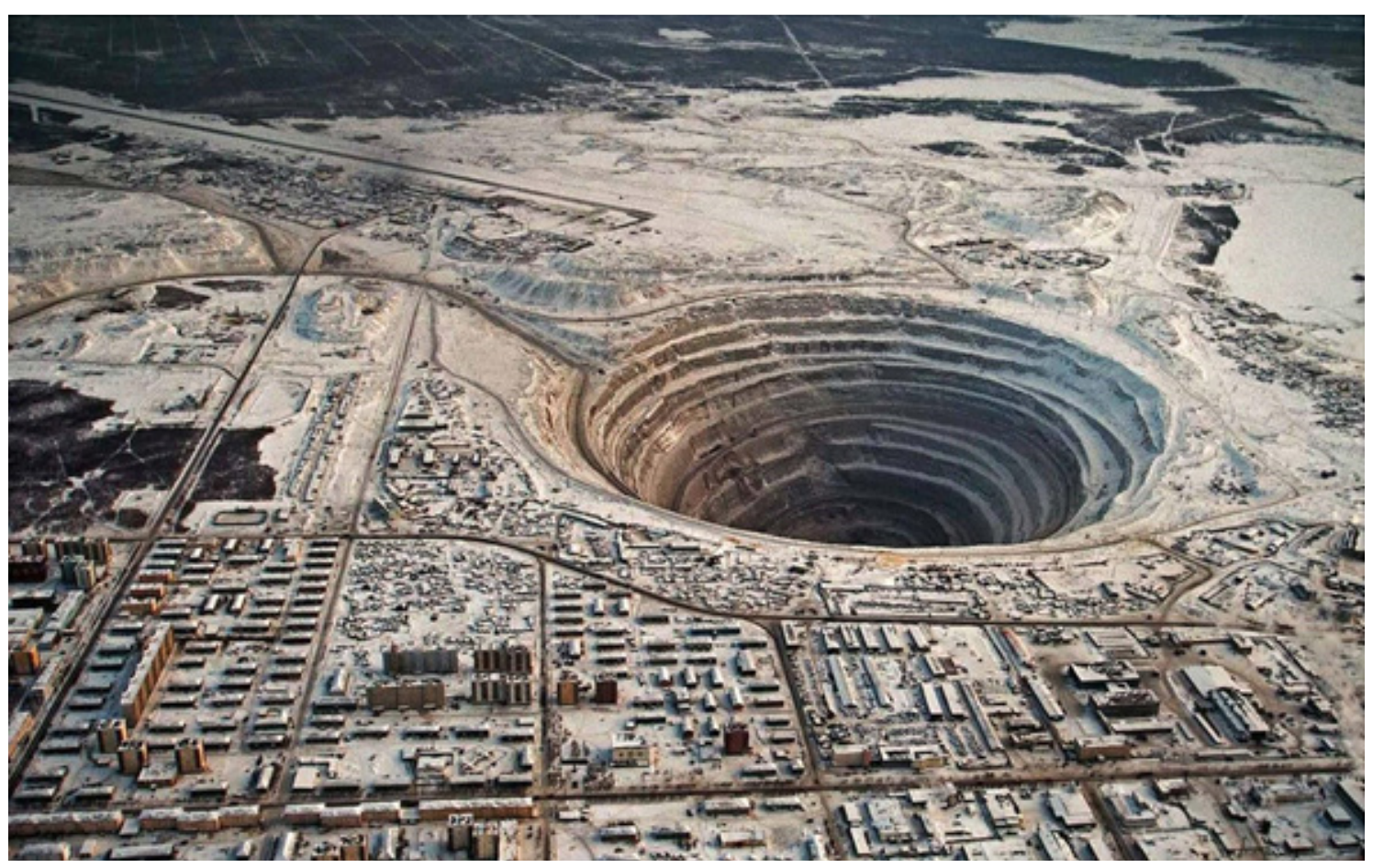

Figure 1: Mirny diamond mine in Eastern Siberia, Russia

Source: Science Source.

\section{Improving resilience to material scarcity with biological models}

Learning from nature and biological formations has become a meaningful and crucial approach for contemporary architects, especially for those who foster a future that does not compete with nature but coexists with it. As a response to the natural disasters worldwide, especially those geologically associated such as tsunami and earthquakes, which have proven their destructive power over the current built environment, architects and structural engineers have found in biomimicry an ecological approach in order to improve future disaster resilience of the buildings. 
In his dissertation Bio-Structural Analogues in Architecture, Joseph Lim (2009) explains that 'the central to the development of architectural concept and design strategy is a form of technological thinking which drew inspiration from other forms of knowledge'. Scientific insights in the technological solutions promoted by nature and their links to architecture has led to a bottom up approach for more resilient structural design. As D'Arcy W. Thompson (1945) wrote, every form in Nature is essentially a product of a set of forces acting on it. This technological feature of the living structures is a parameter that defines the level of resilience of their morphology, since their tessellation grows in intrinsic relationship with the ecosystem and the natural flows within it.

To overcome the problem of scarcity of resources, this paper focuses on describing an approach to material minimization in constructions, based on the investigation of cellular solids as a tectonic system in architecture that is based on a complex form articulation. In particular, this works is inspired by the hierarchical structure of bones as the most prominent example of lightweight and at the same time structurally efficient natural systems. Bone internal microstructure can be used as role model to conceive larger scale constructions, by means of computational design and Additive Manufacturing (AM).

\section{The efficient design of the trabecular bone}

When modern man builds large load-bearing structures, he uses dense solids: steel, concrete, glass. When nature does the same, she generally uses cellular materials: wood, bone, coral. There must be a good reason for it (Ashby 2000).

Natural organisms are based on coherent development of form, material and performance. Cell, as a basic construction unit and a fundamental form of life, has been widely explored since Robert Hooke discovered it and described it in his seminal work Micrographia (Hooke, 1665). Cellular structures in nature can be found in various configurations and scales. The different level of connectivity of cellular structures (topology) is the main parameter that influences the behaviour of the material or structure.

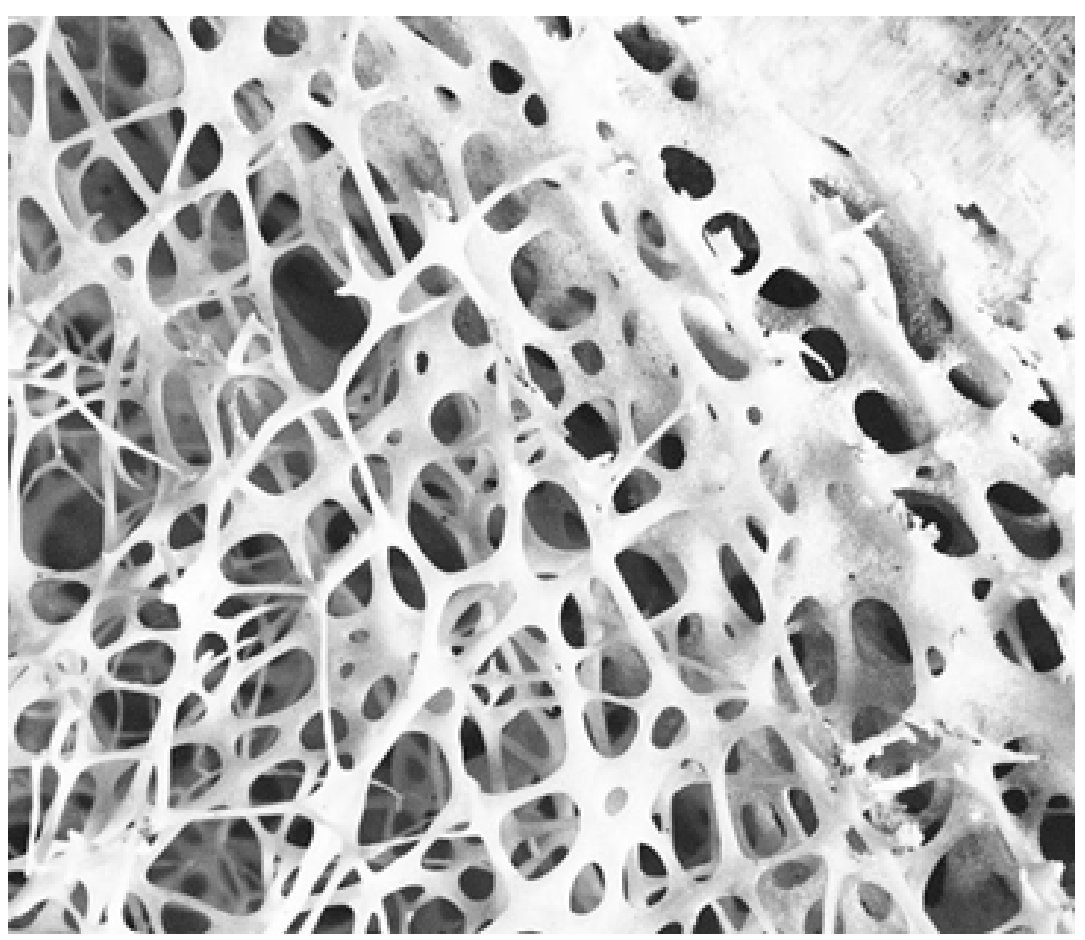

Figure 2: Type of of cellular structures found in nature: Open cell structure in cancellous bone

Source: Science Source. 
The high structural efficiency of the bone structure in particular relies on the internal microstructure (cancellous bone) (Fig. 2), based on cells named trabeculae, that are formed through an iterative and homeostatic load-responsive process. Here, an emergent latticework of fibers constitutes a cellular microstructure informed by its loading conditions, which varies in porosity and in orientation to align with the main stress trajectories to withstand both tensile and compressive forces (Benyus, 2002) (Fig.3).

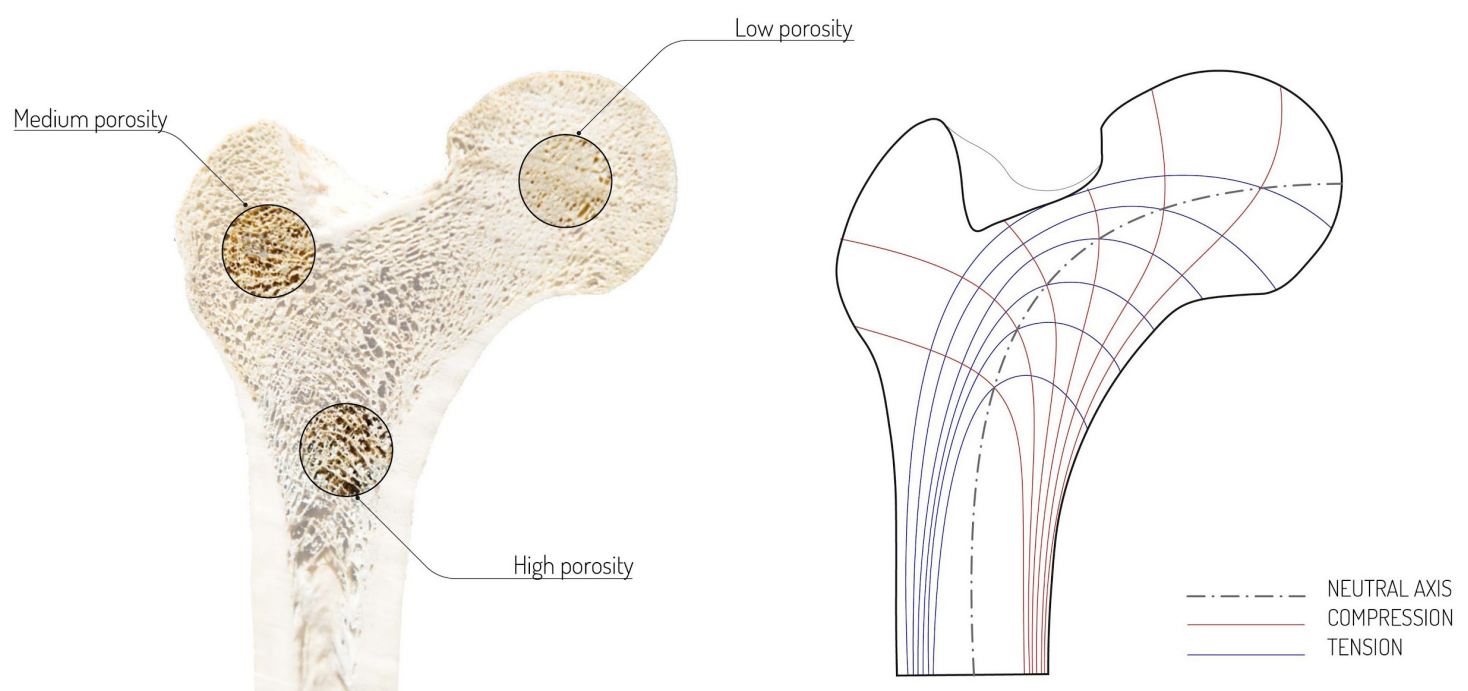

Figure 3: Section of a human femur bone showing degrees of porosity according to a load-responsive material organization

Source: R. Naboni - CREATE Group.

Figure 4: Algorithmic interpretation of the bone remodeling process

Source: Adapted from J.S. Turner, 2012.
The process of bone's structural reconfiguration is called Bone Remodelling Process. Bone remodelling process is a continuous change and adaptation of the trabeculae based on the external loading conditions that are encountered within one's life. More specifically, this process is subjected to the simultaneous action of two types of cells - osteoclasts and osteoblasts, that are evaluating local strain values within the trabecular structure of the bone and adding or removing material accordingly. High strain levels shows that the bone is weaker than desirable and osteoblasts will compensate by adding material in order to reduce strain. Analogously, excessively low strain levels show an unneeded over-mineralization, and the need for osteoclasts to remove material. The balance between these two processes therefore provides a converging point where function and structure are optimized (Turner, 2012). This specific formation process can be synthesized in an algorithm (Fig. 4) which constitutes the procedural base for the generation of the load-responsive cellular envelope tackled in this paper.

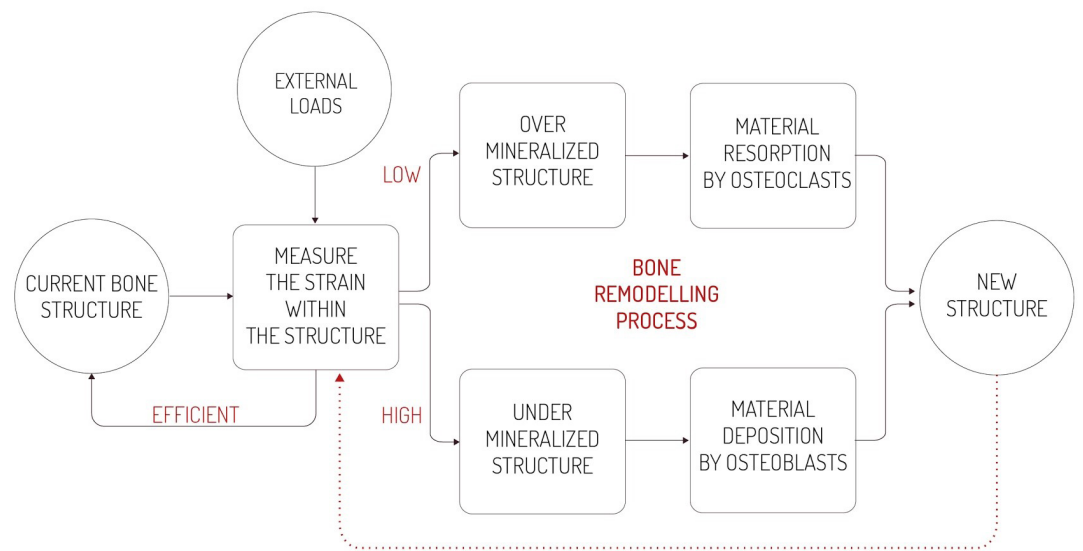


An experimental skin system based on the lattice structure is implemented through the combined use of advanced computational design tools and Additive Manufacturing. Fundamentally, lattice structures are composed of an interconnected network of struts, pin-jointed or rigidly bonded at their connections (Ashby, 2005).

\section{METHODOLOGICAL PROCEDURES}

\section{Computational workflow}

Specific logics referring to the process of bone remodelling such as informed variation of porosity, relative density and trabeculae orientation in relation to main stress trajectories are relevant for this study and implemented into a computational workflow for the design, optimization and fabrication of the so called Functionally Graded Trabecular Tectonics (Fig. 5). As in nature, the goal here is to provide a general methodology for sustainable materialization that is possible to be applied to an infinite variety of formal and loading conditions. This involves the elaboration of a customized computational workflows for the generation and materialization of load-responsive lattice structures. Analogous to the bone remodelling principles, an algorithm performs an iterative mechanical analysis of the overall form with the use of Finite Element Methods (FEM) to compute a highly refined material distribution of free-form building envelopes. The resulting layout is translated in an optimized lattice structure which, in common with the bone trabeculae, varies in porosity, topology and orientation according to the local stress values, and in size to best fit specific shape features. The main input parameters of this process are material properties and fabrication constraints of AM, overall geometry and loading conditions. Variations in any of these would generate different outputs. Morphological, material and performance information is read, analysed and modified iteratively.

What is a particular advantage of this approach is the possibility to be applied to a wide range of materials. Certainly, the mechanical properties of cellular structures are governed by those of the solid materials that they are made of, yet the most influencing parameter is their relative density and topological configuration. More precisely: form does matter, more than material. For this reason, the research focuses on the fabrication method of Fused Deposition Modelling (FDM) with thermo polymers - an additive process that ensures high formal complexity and control over material organisation, relative geometrical freedom and the use of weight and cost effective materials. This naturally profits from the fact that thermoplastic polymers can be modeled into any desired shape while preserving almost entirely their mechanical and thermal properties.

\section{Fabrication Setup}

FDM Printers share common features and characteristics, however, professional applications of Additive Manufacturing are nowadays necessitating more and more specialization in terms of materials, design for fabrication and printer typology. Cellular constructions require discontinuous printing. While this technique is favouring the reduction of material usage, it is highly impacting on the printing sequence, where extrusion has to be continuously retracted and paused, with a large number of travels without material deposition. Typically, this condition is considered inefficient as the printing head is activated and deactivated. In spite of this, a clear advantage of this type of printing is that components are not influenced by warping. This happens when plastics cool down after the extrusion, and temperature gradient in the printing chamber are causing shrinkage. In this case, the reduced area/volume ratio of the components, and their porosity, eliminate completely this phenomenon - making it ideal for the use with any material. 


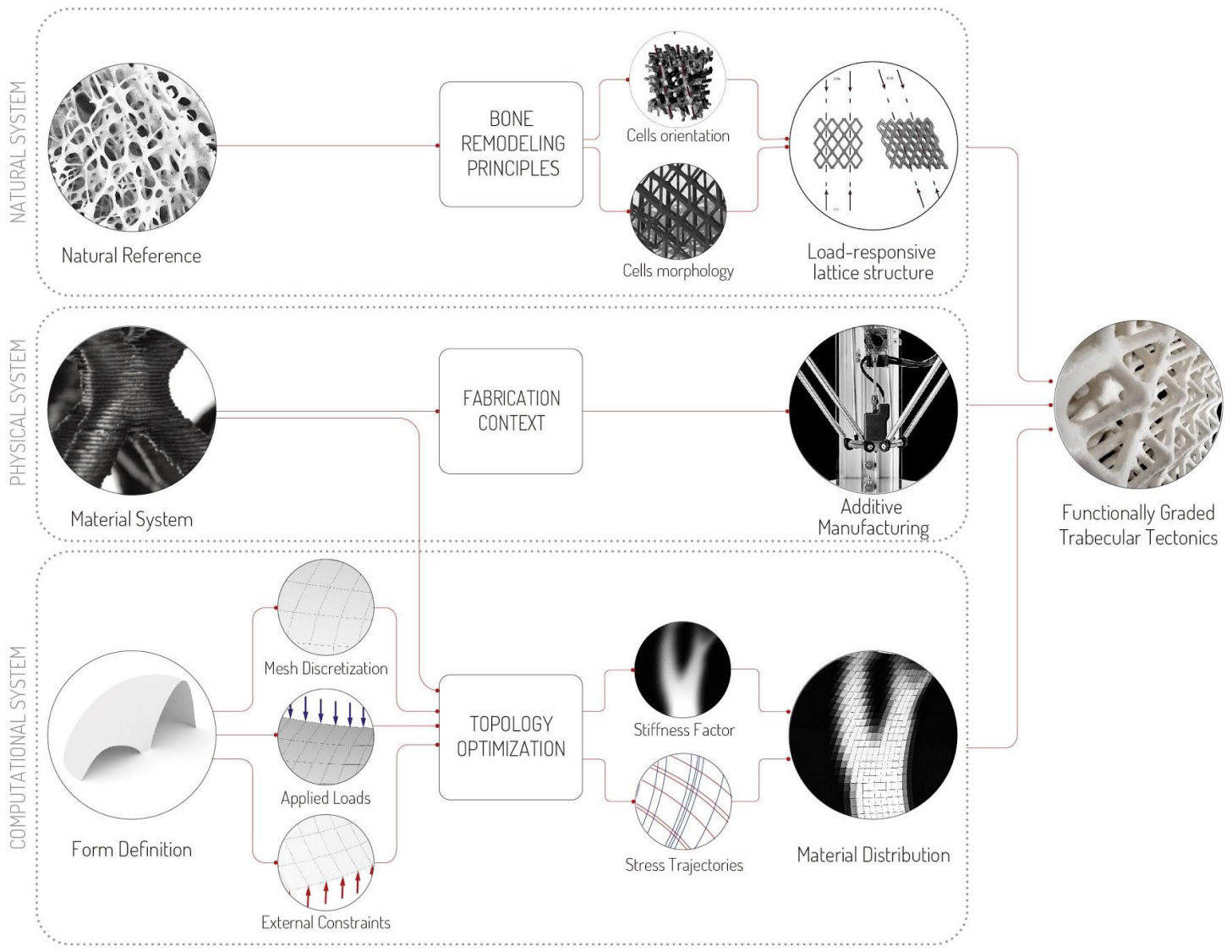

Figure 5: Scheme of the overall design workflow, involving computational tools, fabrication procedures and material information

Source: R. Naboni CREATE Group.

Figure 6: Printing process of the lattice structure by Delta Wasp robot

Source: R. Naboni CREATE Group.
After several tests with different printers, it became evident that Delta Printers are an ideal match for this morphologies: because of their mechanics with minimal mass they are intrinsically, thus permit extreme travel speed for the production of discontinuous geometries such as the lattice structures. In this research, a new type of extruder with a larger nozzle of $1.2 \mathrm{~mm}$ that allows for larger shell thickness without the need of multiple extrusions per layer (Fig. 6).

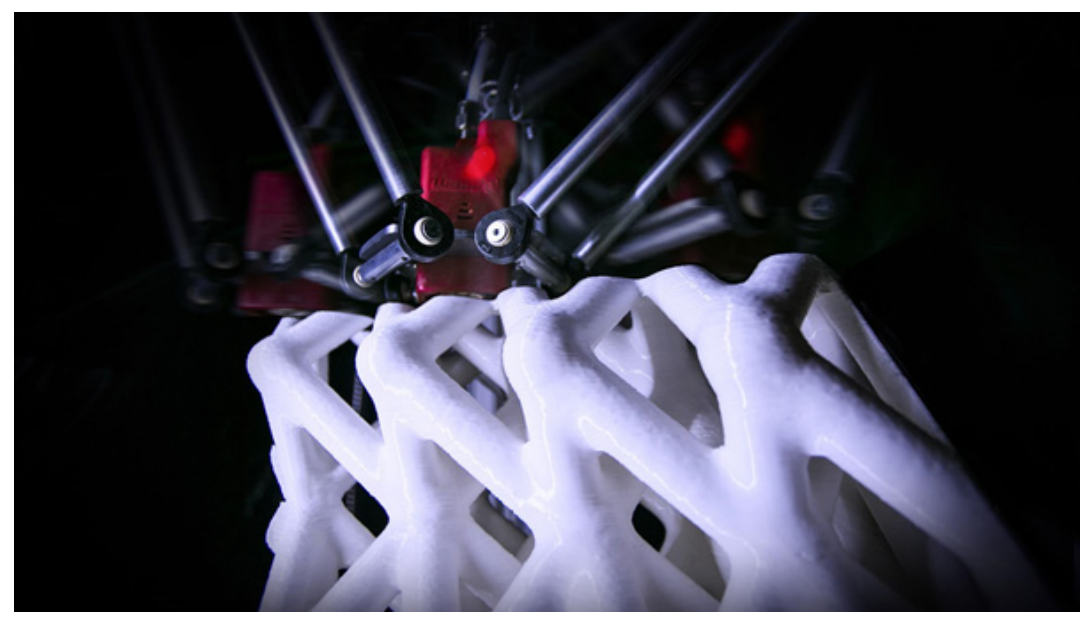


The employed material is High Performance, biodegradable polymer with discrete mechanical properties which are leveraged by its superior printability. An extensive campaign of fabrication tests have been conducted with it to define print settings in relation to geometric constraints, printing time, printing resolution and mechanical resistance of the lattice microstructure. Material characterization was developed mainly through the tensile testing of custom made specimen that resembles the thinnest strut of the lattice structure i.e. diameter $10 \mathrm{~mm}$ with the hollow section of $1.4 \mathrm{~mm}$ thick walls (Fig. 7; Fig. 8).

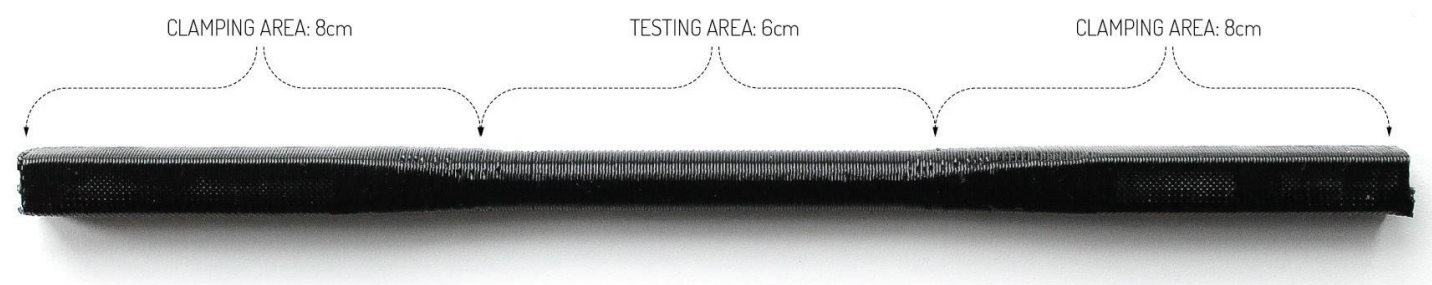

Figure 7: The scheme shows dimensions and geometry of the testing specimen

Source: R. Naboni CREATE Group.
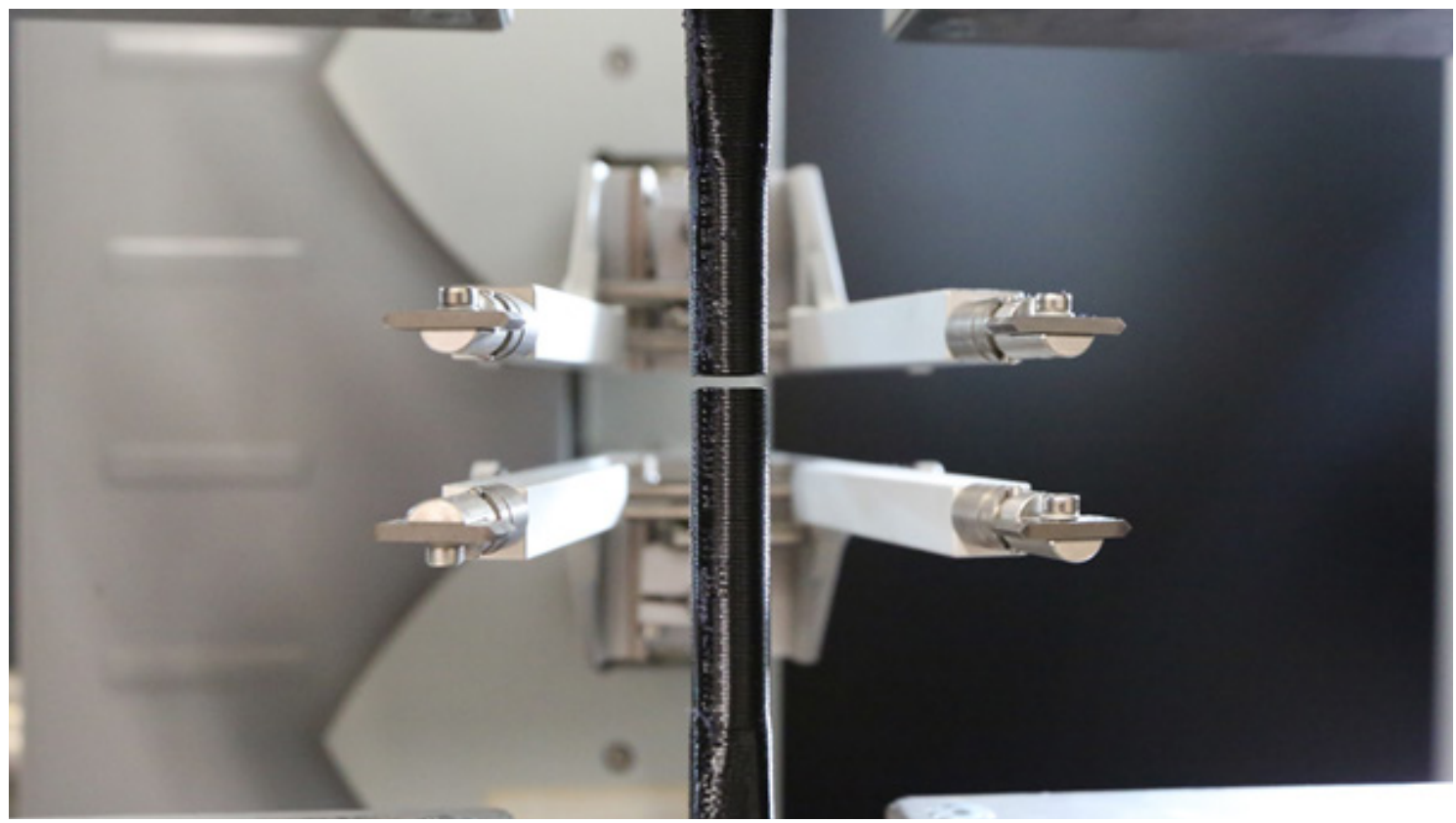

Figure 8: Tested specimen in tension after breaking

Source: R. Naboni CREATE Group

Among various aspects, an important one emerged in the necessity of evaluating models to be printed according to geometry limitations in overhanging angles, to avoid the need of support geometries with consequent inefficiency in the use of material. The relation between the deviation angle from the vertical axis and the number and thickness of shell elements is fundamentally driving the resolution and refinement of the production. 


\section{Study on lattice cell typologies}

As learnt from biological role models, cell is a basic construction unit and it plays the main role in the definition and characteristics of any cellular structure. In nature this is direct expression of material system, which accommodates the biological and mechanical needs of an organism. This implies that previously discussed geometrical constraints of FDM are the first to be taken into account in the critical evaluation for best structural and design solution. A comparative multicriteria analysis of typical three dimensional lattice cells is conducted to evaluate parameters such as printability, relative density and visual permeability. Eight typologies are analysed and shown in Fig. 9, each specimen being bounded in a $10000 \mathrm{~mm}^{3}$ cube with all struts having same cross section diameter of $10 \mathrm{~mm}$.

For what concerns geometrical constraints and evaluation of overhanging angles, a critical threshold is set at $65^{\circ}$ angle deviation from the vertical axis, as this has been established in some of the previous test and studies on printability with basic settings. All the angles below this value guarantee production speed and quality, whereas larger angles than this can be problematic. This problem becomes always more obvious as the printing layer height increases. From the eight evaluated cells, the octahedron (D) and diamond (E) cells show optimal features for FDM fabrication process as they have no horizontal elements and the angles between the struts can be kept out of critical range. In the Fig. 9 red color represents the critical struts.

In the observations regarding relative density values, large differences are highlighted ranging from the very low ones of 0.10 and 0.18 in the case of diamond (E) and (D) octahedron respectively, to the high values of 0.50 as in the case of octet (F) and tesseract (C). The higher relative density means more stiff structure as more than the half of a bounding box is occupied by the cell struts, however, the structure gets heavier and less permeable.

Last, but not least visual permeability is evaluated in respect to the projection of the unit cell on a vertical plane using a $30^{\circ}$ angle of view. This analysis shows again very strong differences among the samples, with the dimension of projected areas ranging from $4900 \mathrm{~mm}^{2}$ to $12100 \mathrm{~mm}^{2}$. Considering the projected area of the bounding box itself that results in 16 $600 \mathrm{~mm}^{2}$, the octahedron with its area of $4900 \mathrm{~mm}^{2}$ obstructs about $1 / 3$ of the visual field, while the octet cell with its projected area of $12100 \mathrm{~mm}^{2}$ blocks around $3 / 4$ of the view.

Everything examined and summed up, octahedron cell has shown to be the most appropriate to guarantee a streamlined production while offering a degree of freedom allowing variable mechanical and visual features.

\section{Constructive Prototype with Octahedron Cells}

Scaling up towards the larger construction components, a further step was undertaken in order to evaluate stiffness, lightness and permeability at full scale according to variation in porosity (relative density). Larger samples of cellular structures have been manufactured inscribed within a $500 \mathrm{~mm}$ wide cuboid component (Fig. 10). Interestingly, same (or close enough) relative density can be reached with different cells size, and very different visual perception. Various configurations have been considered seeking for the geometrical limits and constraints of the lattice structure considering relationship between the cell size, strut thickness and cell angle.

While first experiments involved only regular geometries and standardized angles, further experiments went on testing doubly curved geometries (Fig. 11), cell deformation boundary conditions and varying material distribution. What was observed from these material experiments is that the critical angle of lattice cell is ranging from $45^{\circ}$ to $75^{\circ}$ from horizontal axis (printing plane). A $1 \mathrm{~m}$ tall and $0.5 \mathrm{~m}$ wide doubly-curved component was printed in order to verify such findings. Moreover, this component dimension was considered as optimal size for the building skin that is assembled out of larger number of lattice based components. 


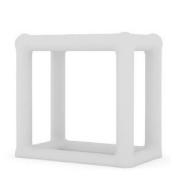

A

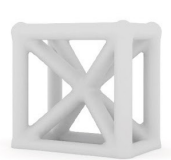

B

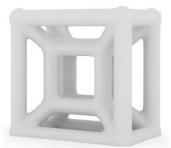

C

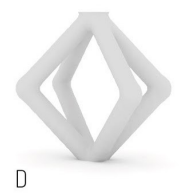

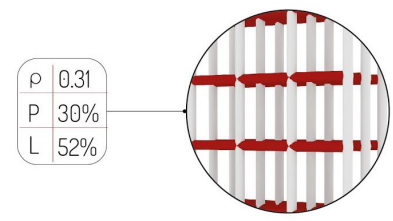
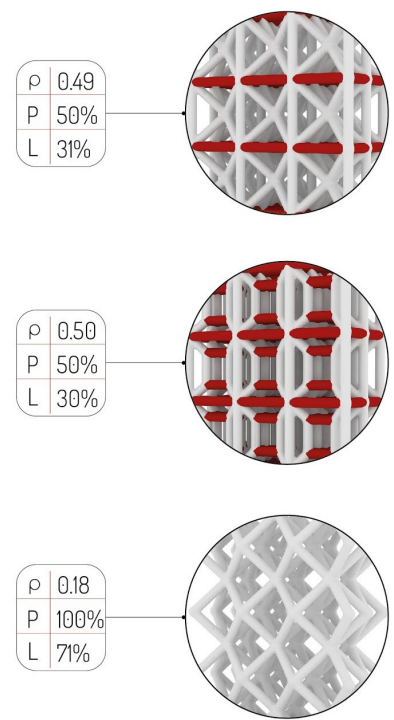
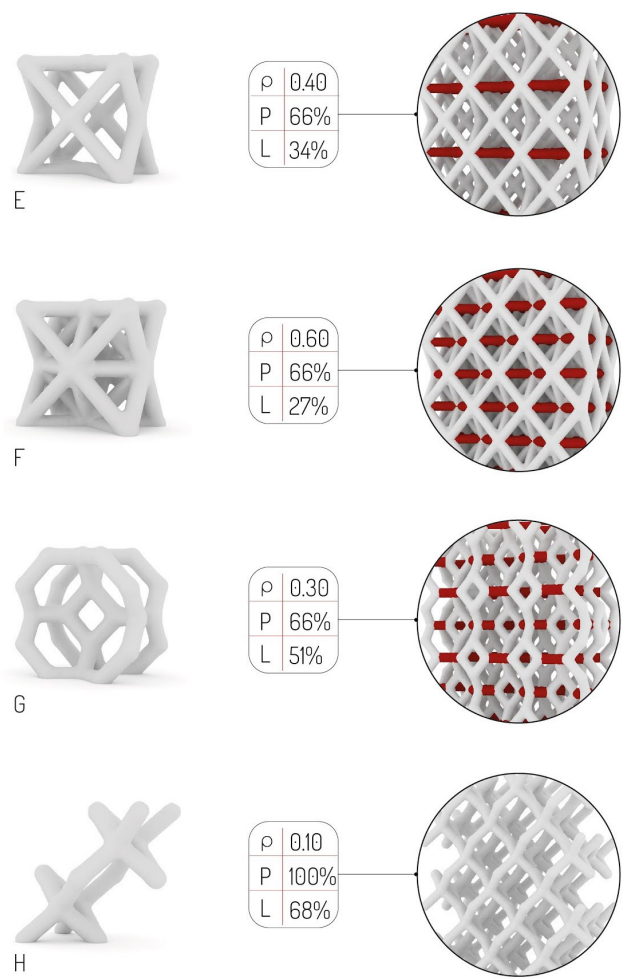

Figure 9: The image shows eight different unit cells typologies for the Cellular Lattice Structure and their observed characteristics; first column shows unit cell types: A - orthogonal grid, B - star, C - tesseract, D - octahedron, E - cross, F - octet, G - vintiles and $\mathrm{H}$ - diamond; second column shows the relative density $(\rho)$, printability $(P)$ and light permeability ( $L$ ); third column shows the repeated unit cell in a skin system, highlighting in red elements that are not possible to be fabricated with FDM.

Source: R. Naboni - CREATE Group.
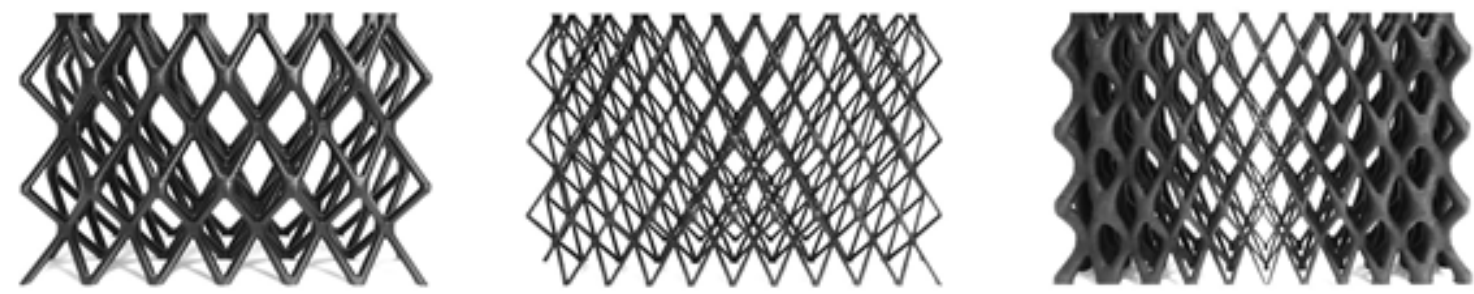

Figure 10: Constructive Prototype: Regular geometry components $500 \mathrm{~mm}$ wide with different relative density: from left - $\rho=$ $0.04, \rho=0.05, \rho=(0.04$ to 0.06)

Source: R. Naboni - CREATE Group. 
Figure 11: Constructive prototype: Doubly-curved component 500 $\mathrm{mm}$ wide and $1000 \mathrm{~m}$ tall

Source: R. Naboni - CREATE Group.

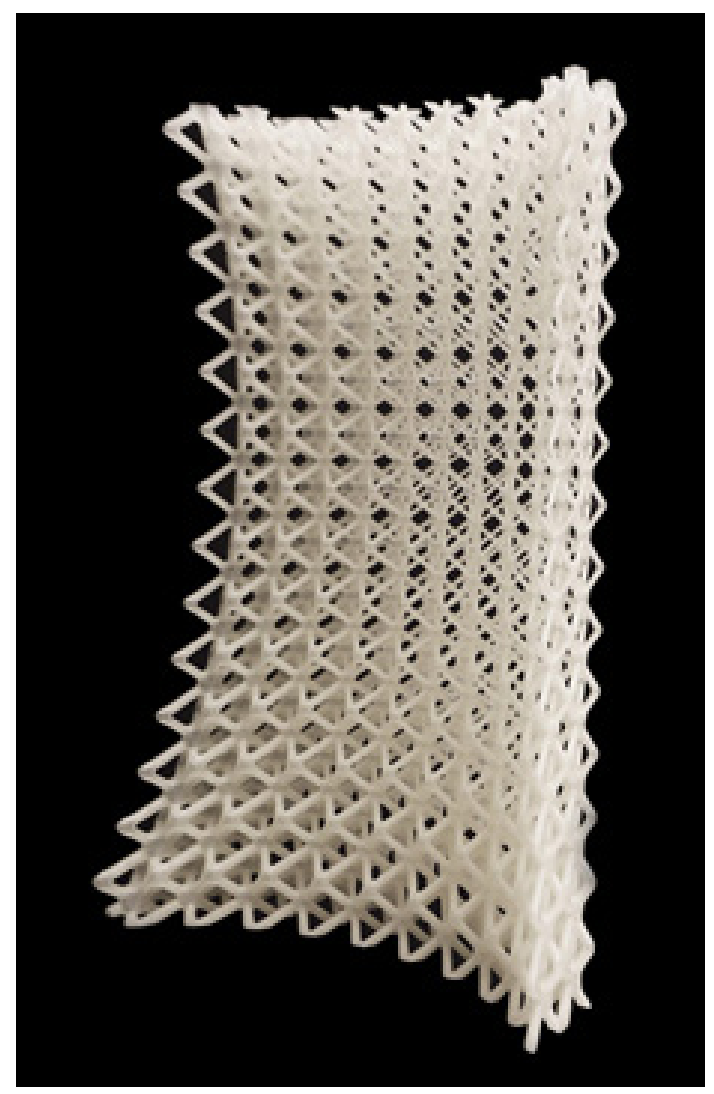

\section{Mechanical Performance of Octahedron Cells}

Consequently, a lattice brick with evaluated octahedron cells was tested in compression in order to measure its mechanical properties and structural behaviour (Fig. 12). The total weight of the brick was $185 \mathrm{~g}$ with the configuration of $2 \mathrm{x} 2 \mathrm{x} 2(\mathrm{x}, \mathrm{y}, \mathrm{z})$ cells. The cell struts were designed under the angle of $60^{\circ}$ and hollow cross section with the wall thickness of $1.4 \mathrm{~mm}$. The test results shows compression resistance up to $3000 \mathrm{~N}$ load, about 1.600 times the weight of the structure.
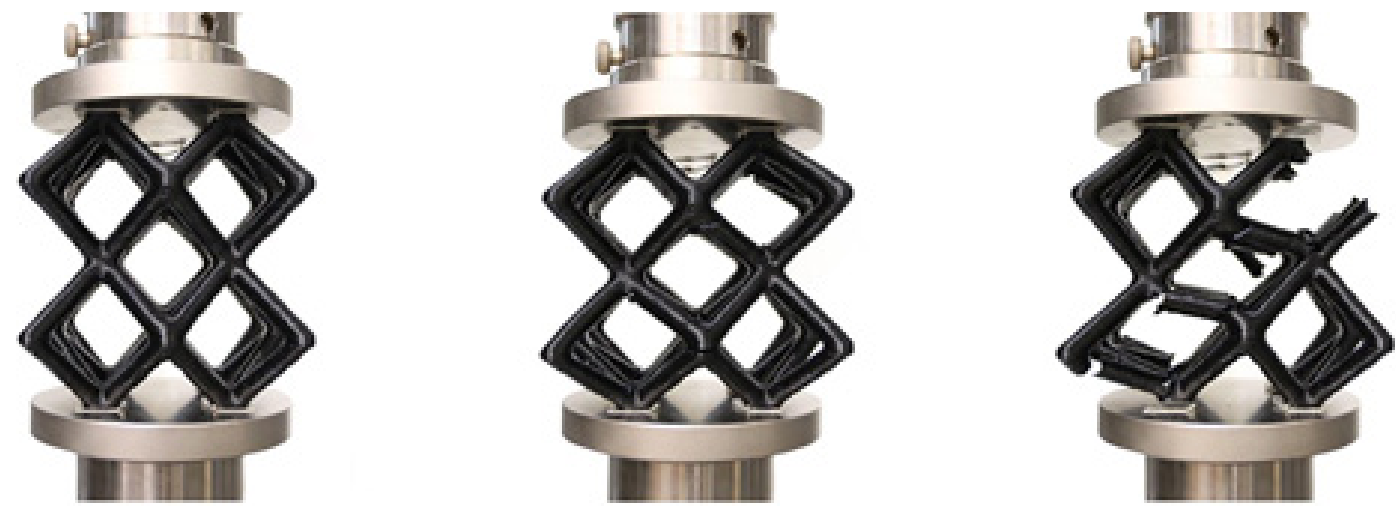

Figure 12: Compression testing of the lattice bricks with octahedron cells

Source: R. Naboni - CREATE Group. 
In the performed tests, the design of the cellular structure extends till the level of the individual layers in such a way that the layer thickness is not used equally along the various part of the structure. Different concurrent optimizations are provided to increase structural performance and improve printability of the cells. The main one is related to the anisotropic nature of 3D printed parts. Anisotropy is not to be considered necessarily as a problem, however, FDM has the constraint of printing with horizontal parallel layers. Since the optimization that involves varying layer orientation according to the orientation of the principal stresses is not possible, to compensate the fact that in certain part of the structure the layer orientation might be inconvenient, it is introduced a correction of the layer thickness in function of the inclination of the printed parts (Fig. 13). This helps either to improve the quality of printing when geometries are oriented with deviation angle from the vertical direction and, moreover, it is reinforcing the tensile and compressive capacities - essentially compensating the anisotropy of the material.
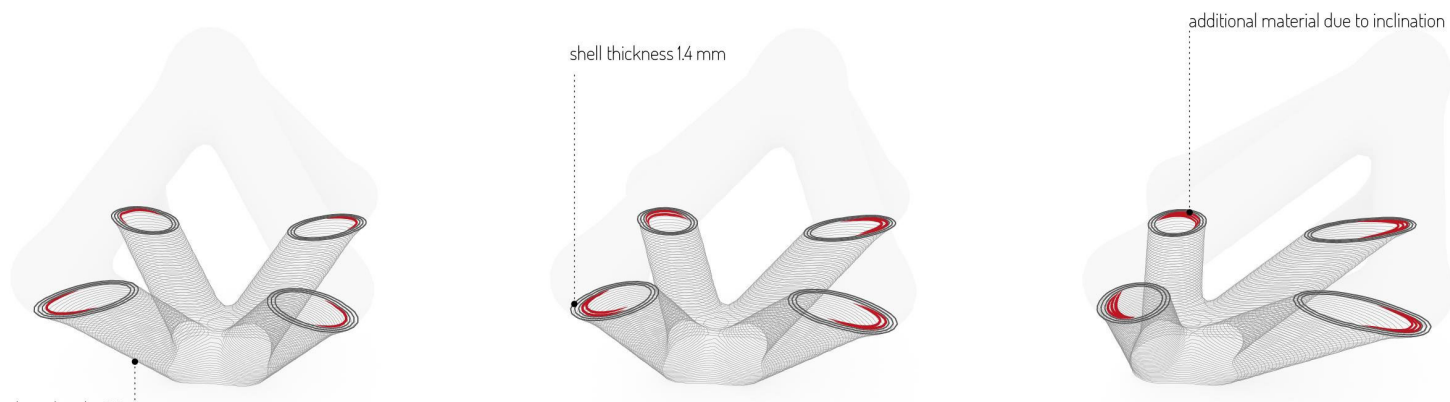

layer height $0.5 \mathrm{~mm}$

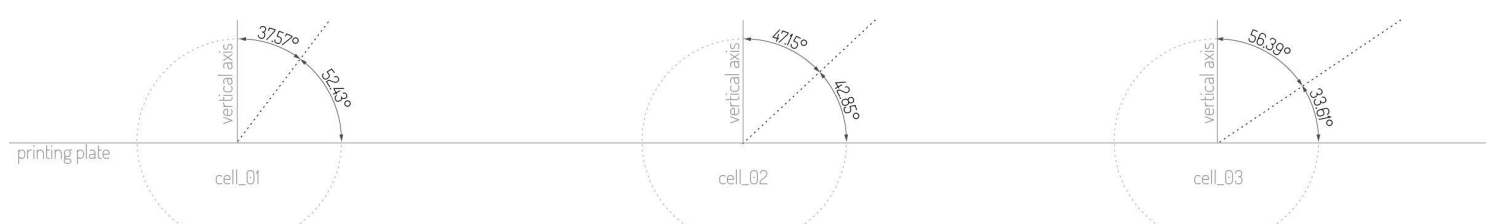

Figure 13: The scheme shows a correction of the layer thickness in function of the inclination of the printed parts

Source: R. Naboni - CREATE Group.

\section{Design and fabrication of Functionally Graded Trabecular Tectonics}

As discussed earlier, the Functionally Graded Trabecular Tectonics is a lattice-based structural system whose main characteristic is the ability to adapt and reconfigure based on variable loading conditions aiming at reaching the most optimized solution for the structural resilience. Similarly to the self-reconfiguration of the bone's trabecule, the lattice structure is defined in response to the stresses that are acting upon it, and therefore it can be called a load-responsive structure. The whole process of lattice generation and optimization is developed within the computational environment, through the set of algorithms tackling the geometrical, structural and fabrication constraints and seeking for the optimal solution that comprises all the parameters involved.

The use of Topology Optimization algorithms allowed the anticipation of the structural performance for different situations of mechanical changes and loading conditions. Most often TO is only used as a preliminary design tool which provides the designer with an insight into efficient structural layout for previously specified boundary conditions. Nevertheless, in this study the final TO output is used to directly inform the generation of a continuous lattice structure. 
The TO is fed with two-dimensional free-form shapes which stand for a 'draft' of potential building envelope configurations, along with a description of some typical boundary conditions such as applied loads, support points and material properties. The algorithm is based on a Solid Isotropic Material Penalization (SIMP) method for material optimization and it iteratively computes stiffness values and allocates material along the given form in a multi-phase process which tends to converge to a $0 / 1$ solutions. These are zones with lowest/highest density of material, which are represented with an interpolation of black/white color values respectively (Bendsøe and Sigmund, 2003). These obtained values are directly converted into Functionally Graded Trabecular Tectonics where mechanical behaviours provide the information to evolve a base polyhedron into highly defined cells with locally optimized size and orientation, struts diameter and cross section (Fig.14).

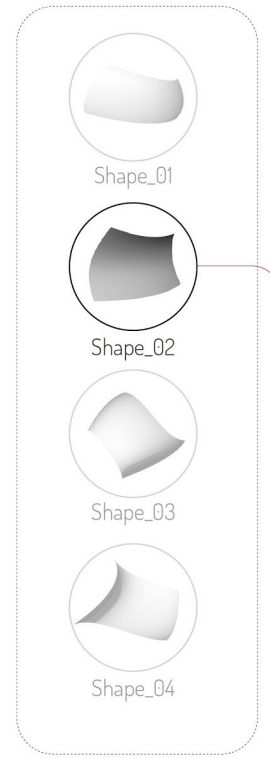

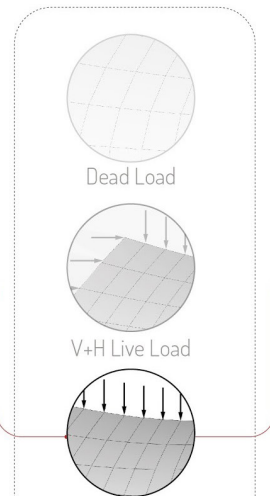
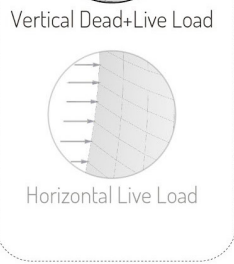
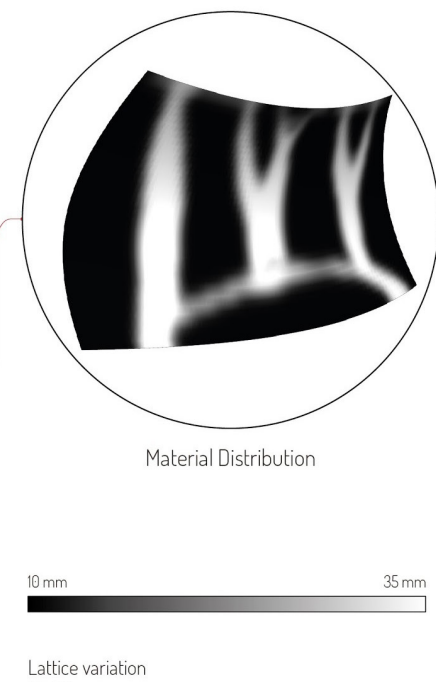

$10-35 \mathrm{~mm}$

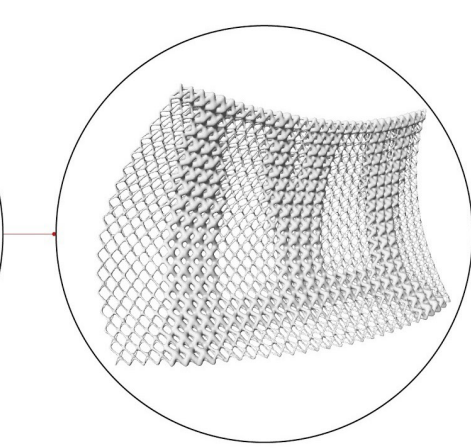

Load-Responsive Lattice Structure

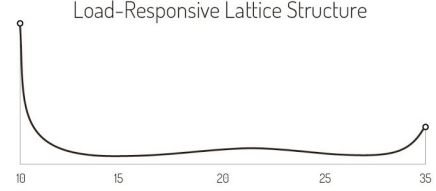

80.000 Struts Diameter Distribution
Figure 14: Workflow of the lattice cellular structure generation from the initial shape definition

Source: R. Naboni - CREATE Group.

Figure 15: Full scale mock-up of a 3D printed Load-Responsive Lattice Structure

Source: R. Naboni - CREATE Group.
Following described computational workflow and methods, and building upon previously developed research on additively manufactured building envelopes (Naboni et, al. 2016; Naboni et, al. 2017), a set of discrete components is fabricated and assembled in a full scale mockup, addressing construction and fabrication aspects of the load bearing lattice structure (Fig. 15).

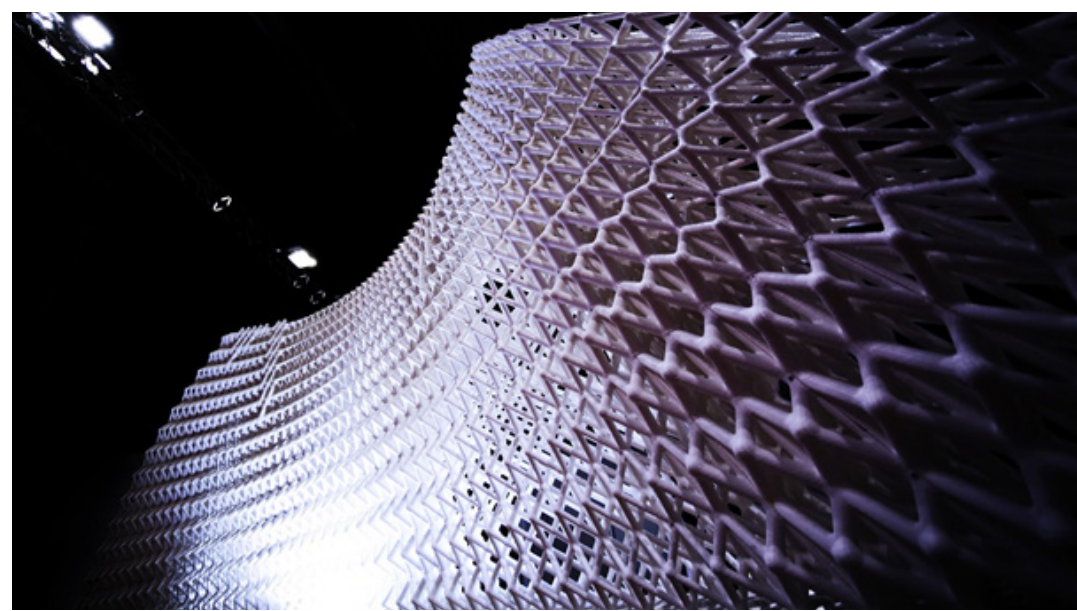




\section{RESULTS}

The research develops a system for free-form building envelopes based on load-responsive lattice structures. The design process is inspired by the formation of cellular bone microstructure, in particular in the creation of gradients of porosity and cell orientation to improve the response to variable loading conditions and foster structural resilience. A novel algorithm-based workflow for the design of complex building skins is introduced. This is articulated in: an iterative procedure for FEM analysis of complex envelope shapes, which suggests optimal material distribution and a process of analysis of FDM material system to define base unit cell for the cellular system. Lattice samples at various scales are successfully prototyped and tested towards the validation of their application in construction. A final full scale mock-up is produced as a proof-of-concept for the load-responsive cellular envelope system, which highlights interesting properties in terms of weight to area ratio, equal to $11 \mathrm{~kg} / \mathrm{m}^{2}$. This is compared with traditional construction skin systems for an intuitive analysis, showing a considerable mass reduction (Table 1). The employed material, High Performance PLA, exhibited ideal characteristics in terms of printability for such geometries, as well as good stiffness and impact resistance.

\begin{tabular}{|c|c|c|c|c|c|c|c|}
$\begin{array}{c}\text { CONSTRUCTION } \\
\text { SKIN SYSTEM }\end{array}$ & $\begin{array}{c}\text { Plastered } \\
\text { brickwork }\end{array}$ & $\begin{array}{c}\text { Curtain } \\
\text { wall }\end{array}$ & $\begin{array}{c}\text { Balloon } \\
\text { frame } \\
\text { construction }\end{array}$ & $\begin{array}{c}\text { Cellular } \\
\text { concrete }\end{array}$ & $\begin{array}{c}\text { Lightweigh } \\
\text { t concrete }\end{array}$ & $\begin{array}{c}\text { Precast } \\
\text { concrete } \\
\text { (hollow planks) }\end{array}$ & $\begin{array}{c}\text { Load-responsive } \\
\text { Cellular Envelope } \\
\text { [mockup] }\end{array}$ \\
\hline $\begin{array}{c}\text { WEIGHT TO AREA } \\
\text { RATIO }\left(\mathrm{kg} / \mathrm{m}^{2} \text { ] }\right.\end{array}$ & 339 & 68 & 73 & 142 & 268 & 36 & 11 \\
\hline
\end{tabular}

Table 1: Weight to Area ratio values of typical construction skin systems in comparison with the realized mockup of Load-

\section{CONCLUSIONS}

Responsive Cellular Envelope

Source: R. Naboni - CREATE Group.

AM methods have undoubtedly introduced novel materialization processes, where logics of sustainability and efficiency typical of mass-production are no longer applicable. Unprecedented control, precision and freedom of this manufacturing allow the conceptualization of unseen architectural systems. Taking inspiration from the remodelling process of bones, a design methodology which adapts to different shapes and loading conditions is developed. The outcome of this process is an envelope system, which allows the creation of light-permeable load-bearing facades with reduced material usage. This experimental approach challenges current design paradigms of lightweight architecture: complex shapes are neither pre-optimized by shape, nor post-rationalized to meet manufacturing constraints. The system has been successfully tested in a laboratory setup. However, its implementation in operative conditions in buildings would require further developments in terms of material and fabrication equipment. This approach can be easily adapted to the use of metal $3 \mathrm{~d}$ printing, to offer a more robust material option at current time. However, the rapid development of thermos-polymers for $3 \mathrm{~d}$ printing with increased chemical, mechanical and weather resistance, offers interesting perspectives of application with FDM. Further development of this work will involve the testing in a relevant environment through a full scale architectural demonstrator, where a wider set of evaluative criteria are to be involved. In the long-time perspective, this research offers an alternative approach to the problem of scarcity of resources by shifting the design towards a higher scale of resolution, inferior to $1 \mathrm{~mm}$. At this scale, nature prefers to operate by articulating material into complex formations to minimize the use of material. With this open perspective, complexity in architecture can be considered as a beautiful opportunity. 


\section{REFERENCES}

Ashby, M.F. (2005). The properties of foams and lattices, Philosophical Transactions of the Royal Society, No.364, November 2005, pp. 15-30.

Ashby, M.F., Evans, A.G., Fleck, N.A., Hutchinson, J.W., Gibson, L.J. \& Wadley, H. (2000). Metal foams - a design guide, Vol. 1, Butterworth-Heinemann.

Bendsøe, M. P. \& Sigmund, O. (2003). Topology Optimization: Theory, Methods and Application, Berlin, Springer.

Benyus, J. M. (2002). Biomimicry. Innovation inspired by nature, HarperCollins Publishers, NYS.

Hooke, R. C. (1665). Micrographia: or Some Physiological Descriptions of Miniature Bodies Made by Magnifying Glasses. London, England: Jo. Martyn, and Ja. Allestree.

Lim, J. (2009) Bio-Structural Analogues in Architecture. BIS publishers.

Naboni, R., Kunic, A. \& Paoletti, I. (2016). Load-responsive skin systems for lightweight architecture. Proceedings of 11th Conference on Advanced Building Skins. Bern, Switzerland. October, 2016.

Naboni, R., Kunic, A., Breseghello, L. and Paoletti, I. (2017). Load-Responsive Cellular Envelopes with Additive Manufacturing. Journal of Facade Design and Engineering. Vol. 5, Special issue Powerskin. November, 2017. Pp. 37-49.

Thompson, D'Arcy W. (1945) On Growth and Form. University Press, Macmillan in Cambridge,New York.

Turner, J.S. (2012) Evolutionary architecture. Some perspectives from biological design, In AD, Architectural Design: Material Computation, No. 216, March/April 2012, pp. 28-33. 